\title{
Dealing with QoE and Power Consumption in Video Distribution Networks
}

\author{
Adrian Popescu \\ Blekinge Institute of Technology, Faculty of Computing, 37179 Karlskrona, Sweden
}

\begin{abstract}
The paper is about the problem of reducing the power consumption in Video Distribution Networks (VDNs) under the condition of best performance provision in terms of Quality of Experience (QoE) measured at the end user. Related to this, it has been observed that, given an end-to-end video distribution network, it is the last networking segment ending to terminal that has the dominant role in the provision of end-user performance. On the other hand, the rest of the video distribution chain can be optimized such to reduce the power consumption under the requirements of provision of specific Quality of Service (QoS) parameters. The paper first provides an overview of VDNs, which is followed by a short presentation of the CONVINcE project. The second part is focused on the problems of performance provision in VDN in terms of best possible Quality of Experience and minimum end-to-end power consumption.
\end{abstract}

Keywords_IP-based video distribution networks; energy saving

\section{INTRODUCTION}

The creation, distribution and delivery of video content is a sophisticated process that contains different elements dedicated to video acquisition, content production and packaging as well as distribution to customers. IP networks are usually used for the transfer of video signals.

Today, the treatment of video content is very complex, and we have a multi-dimensional process, where the most important components are content acquisition, content exchange and content distribution [1].

At the same time, the Internet is today undergoing an adaptation process to provide large demands for bandwidth increase [2]. In this context, one of the most important contributors is the video traffic, which is because of the appearance of new video streaming services like Netflix and Skype-like video communications. This is further complicated because the Internet has democratized the process of creation, distribution and sharing of video like for instance in the case of YouTube. Finally, a very important element is the adoption of new better video formats like the Ultra High Definition (UHD), which was defined and approved by the International Telecommunication Union (ITU), and which needs even more bandwidth [3]. UHD is intended to be used for displays with an aspect ratio of 16:9 and at least one digital input is capable of carrying and presenting native video at a minimum resolution of $3840 \times 2160$ pixels.

To solve the problem of large bandwidth demands and other issues regarding the transition to future Internet (e.g., Software Defined Networking), new architectures have been proposed and prototyped to support a wide range of services, but unfortunately no focus has been given yet to video distribution. This is clearly observed at new suggested architectures like Content-Centric Networks (CCN) [4] and content-based extensions to Software-Defined Networking (SDN) [5]. That means there is strong need for new research and development activities to develop VDNs. Accordingly, a number of important questions must be answered regarding, e.g., future architectures for video delivery with reduced energy consumption, provision of real-time guarantees for live and interactive video streams, subjective and objective metrics for performance measurement, performance optimization [6].

The paper is organized as follows. A short presentation of video distribution networks and the problems associated with them is done in Section II. A short overview of the CONVINcE project is done in Section III. The problem of Quality of Experience (QoE) is presented in Section IV. The problem of power consumption in VDNs is presented in Section V. Connected with this, preliminary elements of power consumption in VDNs is done in Section VI. Section VII is dedicated to a short presentation of alternatives to save energy in Radio Access Networks (RANs). A preliminary solution is suggested in Section VIII for the determination of end-to-end power consumption in a VDN. Finally, Section IX concludes the paper.

\section{VIDEO DISTRIBUTION NETWORKS}

Video distribution networks are distribution networks including elements like encoders, transcoders, multiplexers and decoders as well as networking elements, which are used to support professional video distribution and delivery $[6,7]$. The basic operations are capturing and initial processing of the video content, initial transportation prior to distribution, primary and secondary distribution and finally delivery to end users. Related to this, some of the most serious problems related to video distribution networks is the high energy consumption $[6,7]$.

These networks can be of category terrestrial or satellite or cable television networks. The first category, also known as broadband networks, is based on using the IP technology. 
These networks are targeting individual users although multicast and broadcast mechanisms can turn them into multicast networks as well.

Basically, the video signal is collected at, e.g., a football stadium and transported in compressed form to some broadcast facility that may be placed in another location [7]. The video is then forwarded to a number of secondary broadcast entities for ultimate transmission to end users. Examples of distributions networks are IP based TV networks (IPTV) and Over-The-Top (OTT) networks. Other networks like terrestrial or satellite networks can be also used, but the focus in the paper is on IP-based distribution networks.

An important observation is that today we have a multidimensional process regarding the treatment of video content, where the most important elements are content acquisition, content exchange and content distribution.

Basic operations done on the video signals along the video distribution chain are video coding and compression, encapsulation, forward error correction, transmission, reception and decapsulation, error correction and decompression. Like in the case of other categories of service provider networks, video over IP distribution systems are expected to provide services with a good mix of simplicity, scalability, security, manageability and cost effectiveness. Service Level Agreement (SLA) requirements for video are used to define the service requirements. These are about parameters like network delay, network jitter, packet loss, availability, loss recovery.

The requirements for greening of IP-based video distribution networks heavily complicates the situation. That means one needs to take a closer look at the component elements in the primary and secondary distribution networks and to analyze and develop greening mechanisms used in the particular cases. Major components are access networks (with different categories of technologies of type wired and wireless), core networks, Data Centers (DCs) and storage networks used to provide Web-based services like IPTV, content distribution and cloud-based services.

\section{CONVINCE}

Towards this goal, an important research activity is done in the CELTIC-PLUS project CONVINcE $[6,8]$. This is a three-years research and development project focused on reducing the power consumption in IP-based video networks with an end-to-end (e2e) approach. The project started in September 2014 and it will end in August 2017.

An end-to-end approach is adopted, covering the whole video distribution chain, which starts at the Head End (where the video contents are encoded and streamed) through the networking part (core/metro networks and the access networks) and ending at the (mobile and fixed) terminals, where the content is consumed. A number of 16 industrial and academic partners from 4 European countries (France, Sweden, Finland and Turkey) are participating in the project. Project leader is Thomson Video Networks in France.
A three layers high-level architecture is considered to study the system performance. These layers are low-layer network (containing Data Link and Network layers), Content Distribution Network (CDN) and Video Distribution Network (VDN).

Furthermore, three categories of low-layer networks are considered in the study, which are non-cloud based architecture, edge-cloud based architecture and Software Defined Networking (SDN) / Network Function Virtualization (NFV) architecture.

Four application scenarios are considered in the project, which are Live Video Streaming (LVS), On-Demand Video Streaming (ODVS), Cloud Gaming (CG) and CameraBased Sensor Networks (CBSN).

The research efforts are concentrated on the most important elements in video distribution chain like, e.g., architectures, software and hardware design, protocols as well as basic technologies in terminals. Furthermore, research activities on elements like eco-design, software best practices and power and Quality of Experience (QoE) measurements are done as well.

Good results on energy savings are reported after one year and a half of work, among them $60 \%$ savings at video transcoding and $50 \%$ savings at wireless multimedia sensors [8]. On the other hand, the focus in the second part of the project will be laid on aspects like performance optimization, integration of demonstrators as well as improvements of the performance of individual demonstrators.

\section{QUALITY OF EXPERIENCE}

Quality of Experience (QoE) is a parameter indicating the experience of an end user with the service provided by a particular communication system, which is owned by a particular teleoperator. QoE is basically a subjective parameter, which combines non-technical parameters like user perception, experience and expectations with technical parameters of the particular communication service like, e.g., Quality of Service (QoS) at the application or network layer levels $[9,10]$. In other words, QoE is an indication of how well the system meets the user needs.

It is therefore important that service or network providers understand the existing relationships between QoE and QoS as well as the associated technical parameters.

There are many potential factors that may influence the QoE and they are acting at different levels. These factors can be partitioned into several classes, as indicated in [10]. The most important classes are user-related (e.g., emotion, privacy, expectation), technical (device, application service, network), content-related (content interaction, mobility) and economical (price). This further indicates the complexity existing in defining the appropriate QoE in a particular situation as well as the large number of parameters that may influence it.

Moreover, there are particular parameters that may influence QoS and QoE in different ways like, e.g., in the 
case of Round Trip Time (RTT). Finally, to make things even more complicated, the QoE performance is very likely to look completely different dependent on the particular application like, e.g., in the case Voice over IP (VoIP), video streaming and Web traffic $[9,11]$.

Dealing with QoE in the context of VDN is a sophisticated issue. This is because of the complexity of relationship between QoE and QoS existing at these networks, but also because of the need to reduce the power consumption at VDN, which more or less influences the QoE performance $[6,8]$.

Even more difficult is to define appropriate trade-offs and the associated metrics to relate the parameters influencing the QoE to the parameters influencing the power consumption and efficiency at a network level. For instance, in the case of cellular radio networks, there are two categories of adaptive power schemes related to QoE (or subcarrier allocation schemes) [12]. These are adaptive schemes to maximize the QoE under given constraints on transmission power (or subcarrier resources) and power adaptive schemes to minimize the transmission power or overall network power under given QoE requirements.

Sophisticated optimization problems need to be defined and solved in these cases. For instance, most of the reported solutions solve the optimization problem by using sophisticated algorithms like, e.g., Nash equilibrium, Lagrange multiplier networks [12, 13]. Furthermore, this also demands for studying the relationship between QoE and Energy Efficiency (EE) as well as the associated QoEEE trade-offs. The goal in this case is to design and to study the video distribution networks with reference to the best possible QoE-EE trade-offs, also with regard to the parameters influencing the QoE.

Towards this goal, an interesting definition is suggested in [14], which is called QoE perceived by user per Watt (QoEW). This metric is useful given the difficulties to measure and to model the QoE-EE trade-off for different scenarios and network architectures, where EE stands for Energy Efficiency. QoEW is given by:

$$
Q o E W=\frac{Q o E}{P}
$$

QoEW is defined to be the QoE perceived by users per Watt, which means that, for a given (video distribution) service, a certain amount of power is consumed by a particular user and, based on that, a particular perceived quality is expected to be experienced by the user.

In other words, QoEW measures the QoE at the cost of a particular amount of power. Furthermore, based on that, different optimization studies can be done that combine multiple objectives related to the characteristics of user type and power consumption together with other relevant parameters like network technology, transport protocol, mobile device, battery life, e2e bandwidth and interference factor $[6,10,14]$.

Accordingly, the goal of a so-called Multiple Objective Optimization Problem (MOOP) is as follows [8]:
Minimize energy consumption for a given video session and certain minimum acceptable visual quality

\section{And}

Maximize user QoE for a given video session and certain maximum acceptable energy constraint

Any optimization problem as defined above aims at selecting the best possible combination of parameters in order to provide the expected performance.

\section{POWER CONSUMPTION}

The second important parameter that must be considered at VDN is the power consumption in the e2e video channel, which should be minimized. This is particularly important given the complexity existent in video distribution, e.g., the video data must be transported over a number of different categories of networks (home networks, access networks, core networks) and, moreover, be converted from the electronic domain to the optical domain and back [6].

A good source of information regarding the evolution of energy consumption expected in the next few years in different segments of an operator network is provided in [15]. This data clearly indicates that today the largest energy consumers are the Data Center (DC) and the Mobile Radio Networks (MRN). This observation is expected to become even more accentuated in the future.

Today, the share of the energy consumed by these network elements is in the range of $40 \%$ and $25 \%$, respectively. Moreover, the share of energy consumed by Data Centers is expected to increase even more in the future. The conclusion is that one needs to give a particular focus to energy consumption of these networking elements and, accordingly, to suggest solutions for energy reduction. This needs to be further used in the process of e2e performance optimization with focus on energy reduction and provision of QoE.

Furthermore, the problem of determination of e2e power consumption in a video distribution network is very difficult and today there are no results reported on modeling the endto-end (e2e) power consumption for video flows traversing a (particular) network [8]. This is because of the difficulties existing in collecting information about the power consumption in a network, along the $\mathrm{e} 2 \mathrm{e}$ route. Furthermore, the power consumption in different network elements (e.g., terminal equipment, switch, IP router, gateway, RAN) is a very complex function, which depends upon several parameters, among them, hardware (technology, implementation), software (used for the particular hardware), communication protocol (e.g., IP, IP routing, TCP, UDP), cryptographic algorithms and security protocols (e.g., Transport Layer Security TLS), video distribution system (e.g., IPTV, IP multicast, Data Center) as well as traffic characteristics (e.g., traffic intensity and packet size). It is mentioned that the cryptographic 
algorithms are particularly influencing the power consumption in handheld devices [10]. Ideally, the power consumed by a device in the $\mathrm{e} 2 \mathrm{e}$ chain should be proportional to the offered network load and, accordingly, be defined in form of, e.g., $\mathrm{mW} / \mathrm{Mbps}$, where $\mathrm{mW}$ stands for milliWatts.

\section{DATA CENTERS}

There are two parts contributing to the model for energy consumption in Data Centers (DCs) and accordingly computation of the efficiency in energy consumption. These are [19]:

- System load, which relates to the consumption efficiency of the particular equipment in the Data Center or Telecom Node. This parameter basically represents the ratio IT / networking work capacity available for the given IT / networking power consumption. The system load must be defined as a function of the utilization of given capacity.

- Facilities load, which relates to the mechanical and electrical systems that support the IT / networking electrical load, e.g., cooling systems, air-conditioning units, Power Distribution Units (PDUs), etc.

The general goal at DC is to increase the energy efficiency, which is defined as being the amount of usable energy produced by a machine or device divided by the amount of energy supplied to it.

As a general comment, it is observed that, in general, the IT / networking equipment uses only $30 \%$ of the total energy consumed by a DC [19]. Furthermore, there are a number of so-called DC best practices, which can be used to improve the performance like, e.g., virtualization, dynamic power management and storage consolidation used to improve the IT efficiency. Other practices used to improve the performance of DC are adjusting the processing speed for processors, use of cooling optimization like, e.g., use of blade servers, server virtualization, improved co-location operation, air containment, dynamic Computer Room Air Conditioning (CRAC) unit control, hot/cold aisle isolation, cold aisle pressure control, improved power distribution [19]. With reference to this, solutions based on network virtualization and virtualized DC are of particular interest for CONVINcE.

\section{RADIO ACCESS NETWORKS}

Radio Access Networks (RAN) main functionality is to provide wide-area wireless connectivity to mobile users. Here, the fundamental question is to figure out how to best use and manage the existing limited spectrum to provide the requested connectivity, also under the constraints of acceptable QoE and reduced power consumption. Moreover, RANs suffer because the power consumption to operate and upgrade them is becoming higher and higher also at the price of increased cost. At the same time, the revenue is not growing at the same rate.
Traditionally, the RAN has been considered as being a collection of Base Stations (BSs), each of them acting in a more or less independent way, with decisions on the radio spectrum however with some loose distributed coordination for the purpose of management. Furthermore, the recent developments in form of small cells have created the need for the development of coordinated control algorithms for dense networks. These are algorithms that generally tend to reduce the performance due to diverse limitations [21]. Another problem is because distributed control mechanisms tend to become complex and therefore performance bottleneck [21].

To save energy in wireless networks, sophisticated software systems are used that must be further developed and improved to control the radio systems under the constraints of reduced energy consumption.

The mechanisms developed so far to save energy in RAN are acting at different levels [21]:

- Technology and sub-system level, which means that energy efficient components are used

- Base Station level, where the energy is reduced by directly acting on the Base Station like, e.g., using of sleep mode

- Cell site level, where the energy saving can be done by controlling the activity in the particular cell like the use of greened ad-hoc routing within the cell

- Network level, where the energy can be reduced, e.g., by selectively forcing particular nodes and/or links to sleep down in particular situations

The most interesting and promising techniques considered today in research are $[17,18,20,21]$ :

- Technology and component level, with a particular focus on the power amplifier

- Air interface, which is about increasing the spectral efficiency and decreasing the energy cost per bit

- Base Station adaptation to traffic load, which is about letting the BS enter the sleep mode in the case of low traffic

- Heterogeneous networks, which is about using of small cells that provide energy gains

- Use of advanced architectural solutions

These techniques are today matters for intensive research and organizations like European Union (EU), Celtic, European Telecommunications Standards Institute (ETSI), 3GPP, IEEE and International Telecommunications Union (ITU) strongly support these efforts.

\section{VIII.PRELIMINARY MODELING OF POWER CONSUMPTION}

In the following, the network is modeled as being composed by a number of blocks. A block is defined to be a 
set of sub-blocks or elements with a common general function from the architecture's point of view like, e.g., in the case of wireless access networks there are the following sub-blocks: Radio Base Stations (RBS), system and overhead. The RBS sub-block consumes only a small fraction of the total consumed operational energy, and it depends upon the particular RBS equipment manufacturer, also indicated in $[16,17,18]$. Because of this, the overall efficiency of a Base Station (BS) is generally less than $12 \%$ [18]. The overhead sub-block is about the energy consumed to power different supporting equipment like cooling. Interesting solutions used to compensate for the low efficiency in energy consumption of RBS are in form of, e.g., the so-called system sub-block, which is based on a layered view used to boost up the coverage and the capacity (with the consequence of improving the utilization of radio resources).

To determine the e2e power consumption, the suggested methodology is as follows $[6,8]$ :

- Define the block schemes and the component elements like, e.g., access networks (eNodeB, HGW, ...), backhaul and transport networks (switching, ...), core network (element1, ...) and servers of different categories (Data Center, ...)

- Measure and model the power consumption of each element

- Model and measure the power consumption for the global network in steady state and also when serving passing video flows

The modeling and evaluation activities take into consideration the architectural blocks and the time functioning of component elements as well. Furthermore, it is also assumed that the total traffic coming into the network is equal to the total traffic leaving out of network. A unit of work is defined to be the work associated with the transport of traffic accepted from one external interface to another external interface.

The fundamental requirement is therefore to obtain the minimum possible e2e power consumption under the condition of best possible QoE as indicated by QoEW for the particular architecture.

Finally, a very important effort is regarding the determination of performance optimization algorithms to deal with the minimization of e2e power consumption and, at the same time, to provide best QoE performance at the end user. Towards this goal, it is mentioned that preliminary results have been obtained in CONVINcE, which are reported both in internal project documents and also in publications $[6,8]$.

\section{CONCLUSIONS}

The paper has reported on problems related to dealing with QoE and power consumption in Video Distribution Networks. An important goal in the design of these architectural solutions is to minimize the e2e power consumption and to provide an as good as possible QoE performance at the end user. A good example of such efforts is the CELTIC-PLUS project CONVINcE, with the goal to minimize the power consumption in IP-based video distribution networks, from Head End to terminal.

The first part of the paper is dedicated to overview the main problems related to the design of VDNs with minimum e2e power consumption.

A short presentation of the CONVINcE project is done and preliminary results are reported. Finally, the last part of the paper is about QoE and power consumption in VDNs and the problems associated with this. Preliminary investigation of these issues is done as well.

Future work is about developing, implementation and testing of the optimization algorithms as well as developing of associated theoretical models for CONVINcE. These models are needed in order to support the experimental part of the project, which is about demonstrators of different categories.

\section{ACKNOWLEDGMENT}

This work is supported by the European Celtic-Plus project CONVINcE and was partially funded by Finland, France, Sweden and Turkey.

\section{REFERENCES}

[1] ERICSSON, Video Contribution and Distribution, http://www.ericsson.com/ourportfolio/media-industry/videocontribution-and-distribution

[2] CISCO, "Cisco Visual Networking Index: Forecast and Methodology,2014.2019”, http://www.cisco.com/c/en/us/solutions/collateral/serviceprovider//ip--ngn-ip-next-generation-network/white_paper_c11481360.html

[3] Ultra High Definition Television, International Telecommunications Union, 2012, http://www.itu.int/net/pressoffice/press_releases/2012/31.aspx

[4] Kurose J., Technical Perspective: Content-Centric Networking, Communications of the ACM, Vol. 55, No. 1

[5] Software Defined Networks, http://sdn.ieee.org

[6] CONVINcE: Consumption Optimization in Video Networks, CELTIC-PLUSproject, https://bscw.celticplus.eu/pub/bscw.cgi/d985/CONVINcEstart_lq.pdf

[7] Popescu A., "Greening of IP-Based Video Distribution Networks: Developments and Challenges", $10^{\text {th }}$ International Conference on Communications, COMM 2014, Bucharest, Romania, June 2014

[8] Monnier R., Popescu A. and Ljung R., "CONVINcE: Towards Power-Optimized Video Distribution Networks", $19^{\text {th }}$ Conference on Innovations in Cloud, Internet and Networks (ICIN), Paris, March 2016

[9] Fiedler M., Hossfeld T. and Tran-Gia P.,"A Generic Quantitative Relationship between Quality of Experience and Quality of Service", IEEE Network, March/April 2010

[10] Ickin S.,"Quality of Experience on Smartphones Networks, Application, and Energy Perspectives, $\mathrm{PhD}$ thesis, Blekinge Institute of Technology, Karlskrona, Sweden, 2015

[11] Hossfeld T., Fiedler M. and Zinner T.,'The QoE ProvisioningDelivery-Hystersis and Its Importance for Service Provisioning in the Future Internet, 7th EURO-NGI Conference on Next Generation Internet (NGI 2011), : June 2011, Kaiserslautern, Germany 
[12] Chen L, Wang B., Chen X., Zhang X. and Yang D.,'Utility-Based Resource Allocation for Mixed Traffic in Wireless Networks", IEEE Infocom 2011

[13] Boche H. and Schubert M.,"A Generalization of Nash Bargaining and Proportional Fairness to Log-Convex Utility Sets with Power Constraints", IEEE Transactions on Information Theory, Vol. 57, No. 6, June 2011

[14] Zhang X., Zhang J., Huang Y. and Wang W.,'On the Study of Fundamental Trade-Offs Between QoE and Energy Efficiency in Wireless Networks", Transactions on Emerging

Telecommunications Technologies, Vol. 24, April 2013

[15] US Department of Energy,'Best Practices Guide for EnergyEfficient Data Center Design", http://www1.eere.energy.gov/femp/pdf/eedatacenterbestpractices.pdf

[16] Kharitonov D.,"'Green Telecom Metrics in Perspective,", $18^{\text {th }}$ IEEE Asia-Pacific Conference on Communications (APCC), Jeju, South Korea, October 2012
[17] ETSI-2009, "Energy Efficiency of Wireless Access Networks Equipment", ETSI document, 2009

[18] ETSI-2011, "Environment Engineering (EE) Energy Efficiency of Wireless Access Networks Equipment”, ETSI document, 2011

[19] Minoli D.,'Designing Green Networks and Network Operations: Reducing Enterprises' and Carriers' Carbon Footprint and Saving Run-the-Engine Costs with Green Networks and Data Centers, CRC Press, ISBN 978-1-4398-1638-7, 2011

[20] ETSI-2012, "Technical Specification TS 102706 Environment Engineering (EE) Energy Efficiency of Wireless Access Network Equipment," ETSI document DTS/EE-00007

[21] Hossain E. Bhargava and Fettweis G.P., Green Radio Communication Networks. UK: Cambridge University Press, 2012 